\title{
A New Paradigm For Studying The Economic And Behavioral Consequences Of Framing Health-Related Decisions
}

Irwin P. Levin (E-mail: irwin-levin@uiowa.edu), University of Iowa

Marco Lauriola (E-mail: marco.lauriola@uniroma1.it), "La Sapienza" University of Rome

\begin{abstract}
Traditional attribute framing effects occur when the same object is evaluated differently depending on whether a particular attribute is labeled or framed in positive or negative terms. For example, in one of our earlier studies, " $80 \%$ lean ground beef" was evaluated more favorably and was "worth" 8 cents more per pound than "20\% fat ground beef." In the present study of health-related judgments and decisions, attribute framing effects were extended to situations where consumers had to infer framing valence depending on whether one's health status was described in comparison to a lower standard or a higher standard of reference. For example, a person's health status was rated higher when the same level of vitamin intake was stated in terms of its distance above an established low-protection level compared to when it was stated in terms of its distance below an established high-protection level.
\end{abstract}

\subsection{Introduction}

$T$

versky and Kahneman's (1981) classic "Asian disease problem" sparked considerable interest in "information framing" effects - how the same information can lead to different decisions depending on the way that information is labeled or framed. In the case of the Asian disease problem, respondents were more apt to make risky decisions when options were described in terms of potential losses than when they were described in terms of potential gains. This is known as the "Risky Choice Framing Effect." A somewhat simpler framing effect that doesn't necessarily involve the element of risk is known as the "Attribute-Framing Effect."

An Attribute-Framing Effect is found when the evaluation of a given object or product differs depending on whether a key attribute is described in positive terms or negative terms. Because a single attribute within any given context is the subject of the framing manipulation, attribute-framing experiments provide researchers with a very general and, indeed, flexible paradigm to test how the positive/negative valence of available information influences one's judgment processes.

Experimental studies of attribute framing often involve consumer judgments, including health-related judgments. For instance, Levin and Gaeth (1988) showed that perceptions of the quality and healthiness of ground beef depended on whether the beef is labeled as " $75 \%$ lean" or " $25 \%$ fat." Levin, Gaeth, Schreiber and Lauriola (2002) added an analysis of the economic impact of attribute framing by showing that consumers were willing to spend an average of 8.2 cents more for a one-pound package of " $80 \%$ lean ground beef" than for a one-pound package of " $20 \%$ fat ground beef." Attribute framing sometimes involves the evaluation of risky behaviors. For instance, Van Schie \& Van der Pligt, (1995) showed that emphasizing "potential positive outcomes" rather than

Readers with comments or questions are encouraged to contact the authors via email.

"negative ones" in a risky option led to increased preference for this option. This has important implications for 
health-related decisions. McNeil, Pauker, Sox and Tversky (1982) showed that a medical treatment was more apt to be selected when it was described in terms of success rate rather than failure rate.

After an extensive literature review, Levin, Schneider \& Gaeth (1998) came to the conclusion that the processes accounting for attribute framing effects are associative processes which produce more favorable evaluations when the framed attribute primes positive associations rather than negative ones. We refer to this process and its expected effects as the "standard" attribute framing effect.

In the present study we provide a different and, perhaps more general, form of attribute-framing, where a given attribute is first described relative to a reference point, and then the framing valence is inferred based on whether the distance from the reference point is viewed as a positive or a negative. We define the positive/negative conditions in terms of valence consistent shifts associated with gains and losses relative to higher or lower standards of risk and protection. Thus, describing one's health status as a given level above a lower standard of risk is expected to produce a consistent negative shift in valuation compared to describing one's health status as a given level below a higher standard of risk which is expected to produce a consistent positive evaluation shift.

\subsection{Aims and Scope}

In the present study, we first test the so-called "general" attribute-framing effect by comparing valence consistent shifts across variations in standard of reference. Thus, for example, we expect positive consistent shifts when evaluating one's exposure to risk as being below a higher standard of risk. Conversely, we expect negative consistent shifts when evaluating one's exposure to risk as being above a lower standard of risk. Second, we expand on previous research by comparing this "general" attribute-framing effect in two different health domains: either evaluating one's exposure to risk factors such as high cholesterol or judging one's exposure to protective factors such as vitamin intake.

We administered four different versions of the attribute-framing problem by providing different frames of reference for a health-risk and health-protection scenario. (A, B, C, and D in the figure). We define each condition as representing positive or negative framing, depending on whether that condition represents a particular value as being below a stated high-risk or high-protection level, or as being above a stated low-risk or low-protection level. This represents an extension of earlier definitions of attribute framing.

Condition A describes one's blood cholesterol level as 4/10 above the low-risk level. We define this condition as negative framing because one's blood cholesterol level is described as an increase in risk relative to a low-risk reference point, which is apt to be viewed as bad or negative. Condition B describes one's blood cholesterol level as $6 / 10$ below the high-risk level. We define this condition as positive framing because one's blood cholesterol level is described as a loss of risk relative to a high-risk reference point, which is apt to be viewed as good or positive. We thus expect risk to be rated higher in Condition A than in Condition B.

Condition $\mathrm{C}$ describes one's Vitamin E consumption level as 4/10 above the low-protection level. We define this condition as positive framing because one's vitamin consumption level is described as a gain of protection in comparison to a low-protection reference point. Condition D describes one's Vitamin E consumption Level as $6 / 10$ below the high-protection level. We define this condition as negative framing because one's Vitamin Consumption level is described as a loss of protection in comparison to a high-protection level. We thus expect protection level to be rated higher in Condition $\mathrm{C}$ than in Condition D. 
A)

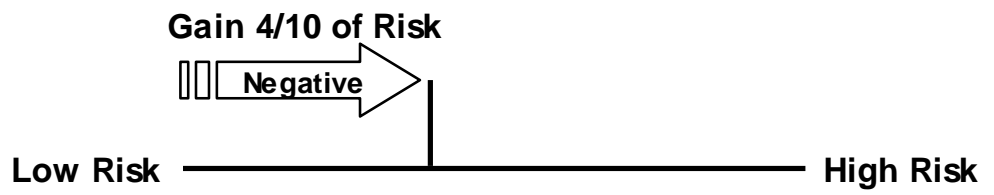

B)

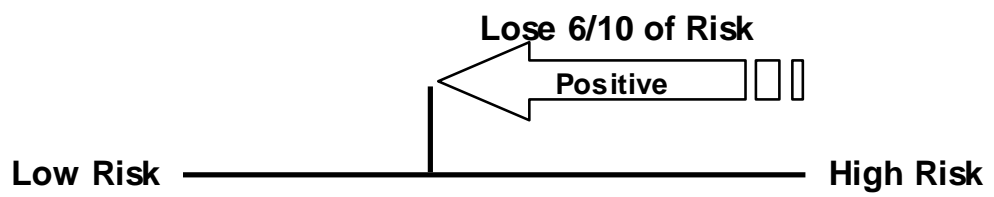

C)

\section{Gain 4/10 of Prot.}

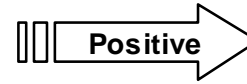

Low Prot.

High Prot.

D)

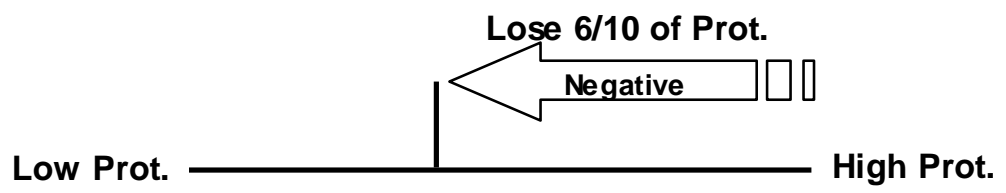

The same blood cholesterol level of say 240 is expected to produce a more favorable attitude when it is expressed in terms of its distance below the high-risk level of 300 (i.e., a risk loss) than when it is expressed in terms of its distance above the low-risk level of 200 (i.e., a risk gain). Conversely, the same objective level of vitamin intake, say $560 \mathrm{IU} /$ day per day, is expected to lead to more favorable evaluations when expressed in terms of amount above some designated low (undesirable) level (i.e., a protection gain) than when expressed in terms of amount below some designated high level (i.e., a protection loss).

\subsection{Method}

\subsection{Participants}

Experiment 1: Forty-two trained undergraduate students each instructed to interview 3 males and 3 females of varying age (20-80 years old) contacted 252 eligible participants, who met the following criteria: 1) having at least a secondary school degree; 2 ) being in relatively good health (i.e., never admitted to a hospital during the past 12 months); and 3) being able to complete a paper and pencil survey. Eligible participants agreed to participate in a larger study of personality and risky health decisions (Lauriola, Russo, Lucidi, Violani \& Levin, submitted for publication). Twelve participants dropped out of the study. Thus, data analyses were completed on 240 cases (118 males, 122 females; mean age 46.99 years; s.d. 19.01 years).

Experiment 2: Thirty-three trained undergraduates contacted 198 eligible participants, who met the same criteria as in experiment 1. Eligible participants agreed to participate in a two part-experiment in which framing experiments were conducted according to a within-subjects design. Seventeen participants dropped out of the study before completing part two of the study. Thus, data analyses were completed on 181 cases (117 males, 64 females; mean age 46.48 years; s.d. 18.96 years). 


\subsection{Research Design}

Experiment 1: Framing problems were constructed according to a $2 \times 2$ design with the framing valence (Positive Vs Negative) and the problem focus (Prevention vs. Promotion) as independent factors. Either the positive or the negative valence condition of each framing type was administered between subjects. Half of the participants completed framing problems about blood cholesterol level, the other half completed problems about vitamin consumption level. Concern about blood cholesterol is clearly related to the prevention of coronary heart disease (CHD), while vitamin intake is reasonably related to general health promotion. Throughout the paper we will refer to these variants of the decision problem as prevention and promotion focused problems, respectively.

Experiment 2: The same framing problems as in Experiment 1 defined a $2 \times 2$ mixed design with the framing valence as a repeated factor (Positive vs. Negative) and the problem focus (Prevention Vs Promotion) as an independent factor. Unlike Experiment 1, the framing valence factor was manipulated within-subjects. Thus, two sessions were scheduled, spaced about two weeks apart, to give each of 181 participants both framing conditions but without their recollection of earlier responses. Information frame is usually manipulated as a between-subject factor to avoid demand effects to appear consistent when responding to identical decision problems. However, Levin et al. (2002) showed that greater statistical power could be achieved without subjects recognizing the identity of differently framed problems when the problems were administered one week apart.

\subsection{Framing Problems}

The prevention focused scenario required subjects to read about a person over the age of 60 whose blood cholesterol level was $240 \mathrm{mg} / \mathrm{dl}$. That person's cholesterol level was either described as being $40 \mathrm{mg} / \mathrm{dl}$ above an established low risk level of $200 \mathrm{mg} / \mathrm{dl}$ or as being $60 \mathrm{mg} / \mathrm{dl}$ below an established high-risk level of $300 \mathrm{mg} / \mathrm{dl}$. That is, the attribute level of $240 \mathrm{mg} / \mathrm{dl}$ was either $4 / 10$ of the range $300-200 \mathrm{mg} / \mathrm{dl}$ above the $200 \mathrm{mg} / \mathrm{dl}$ or $6 / 10$ below $300 \mathrm{mg} / \mathrm{dl}$. Likewise, the promotion focused scenario required participants to read about a person over the age of 60 whose Vitamin E consumption level was 560 IU/day per day. That person's vitamin consumption level was either described as being $240 \mathrm{IU} /$ day below an established high protection level of $800 \mathrm{IU} /$ day or as being $160 \mathrm{IU} /$ day above an established low-protection level of $400 \mathrm{IU} /$ day. The attribute level of $560 \mathrm{IU} /$ day was thus either $4 / 10 \mathrm{of}$ the range $800-400 \mathrm{mg} / \mathrm{dl}$ above the low-protection level of 400 IU/day or $6 / 10$ below the high protection level of 800 IU/day.

\subsection{Dependent Variables}

The attribute being framed in the present study was either the described person's blood cholesterol level (i.e., level of exposure to a risk factor) or the described person's vitamin consumption level (i.e., level of exposure to a protective factor). Research participants judged the described person's exposure to risk or protective factors on each of five ten-step bipolar adjective scales - healthy-unhealthy; good-bad; safe-dangerous; not worried-worried; optimistic-pessimistic, with higher scores indicating a more negative evaluation in terms of either higher perceived risk or lower perceived protection.

\subsection{Results}

\subsection{Experiment 1}

To investigate the effect of design variables on respondents' attribute evaluation, we computed a summated rating of the adjective scales (Cronbach's Alpha $=.93$ with five items) and used this summated rating as the dependent variable in a factorial analysis of variance, with the framing valence (positive vs. negative) and the problem focus (prevention vs. promotion) as independent factors. The omnibus F-test approached statistical significance for the framing valence main effect and for the framing valence by problem focus interaction effect. As shown in Figure 1, a further planned comparison analysis revealed that there was an overall tendency to evaluate that person's health status in a more negative way in the negative valence condition $\left(\underline{\mathrm{F}}_{1,233}=3.29, \underline{\mathrm{p}}=.07\right)$, but that this expected framing valence effect attained statistical significance only in the promotion focus condition $\left(\underline{F}_{1,234}=\right.$ 
$4.98, \mathrm{p}<.05)$. Thus, it is concluded that the negative framing valence tended to produce an overall negative shift in attribute evaluation and that this effect was stronger when respondents judged an established vitamin consumption level.

\section{Experiment 1}

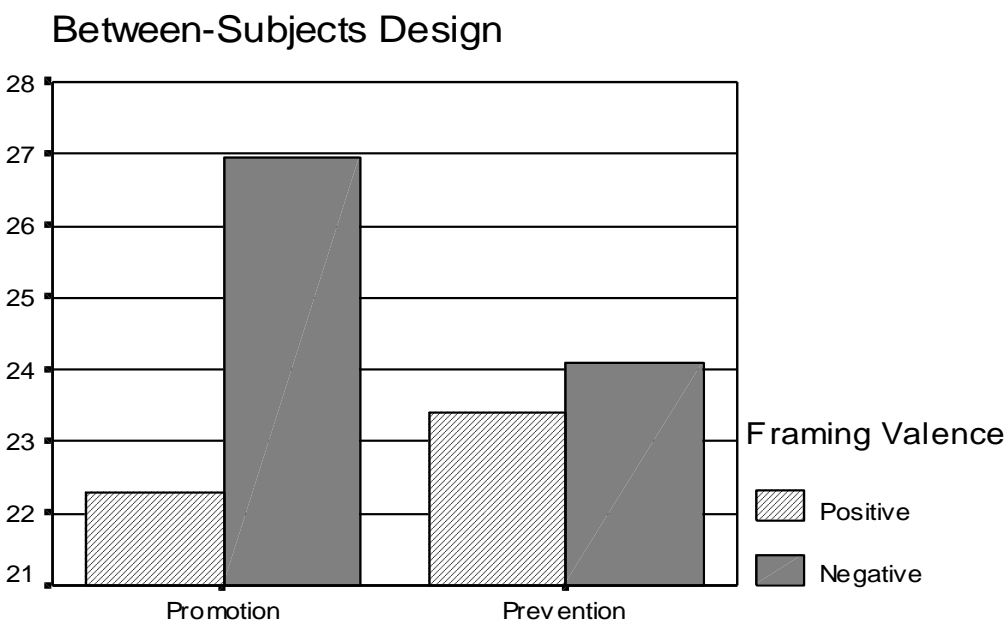

Problem Focus

\subsection{Experiment 2}

We computed a summated rating of the adjective scales used as empirical indicators of the framing valence effect separately for the positive and the negative valence conditions (Cronbach's Alpha coefficients were .95 and .94 , respectively) and used these ratings as levels of a repeated factor in a mixed ANOVA model with the problem focus as the between-subjects factor. The omnibus F-test for the framing valence main effect was again marginally statistically significant $\left(\underline{\mathrm{F}}_{1,180}=3.75 ; \mathrm{p}=.054\right)$, but the average scores reported in Figure 1 as a function of framing valence and problem focus are consistent with our theoretical expectations, as there was an overall trend to evaluate that person's health status in a more negative way in the negative valence condition than in the positive valence condition.

\subsection{Discussion}

In the present study we provided a more "general" operative definition of attribute-framing, where a given attribute was first described relative to a reference point, and then the framing valence is inferred based on whether the distance from the reference point is viewed as a positive or a negative. We then tested this so-called "general" attribute-framing effect by comparing valence consistent shifts when evaluating one's exposure to risk as being below a higher standard of risk (i.e., positive) or above a lower standard of risk. (i.e., negative). A secondary goal of the study was to expand on previous research by generalizing the attribute-framing effect to prevention-focused and promotion-focused health domains. Thus, we compared the attribute framing effect in the domain of evaluating one's exposure to risk factors for health to additional positive/negative valence conditions where respondents evaluated one's level of exposure to a protective factor for health. Two experiments were conducted in which the framing valence was either manipulated between-subjects or within-subjects. 


\section{Experiment 2}

\section{Within-Subjects Design}

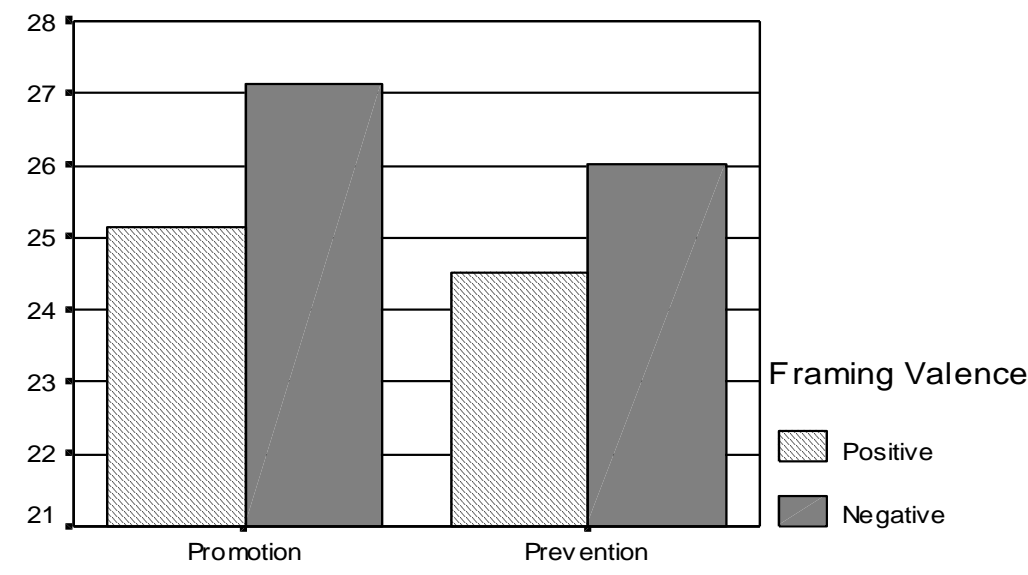

Problem Focus

Overall, our results supported the general hypothesis of the study, as there was a negative bias in evaluating the framed attribute in the negative valence condition, both in the between-subjects and in the within-subjects experiment. This finding supports the appropriateness of the new paradigm for studying economic and behavioral consequences of framing health-related decisions. Although the framing valence effect was smaller and not statistically significant in the prevention focused condition of the between-subjects experiment, the valence consistent shift appears to generalize across prevention-focused and promotion-focused health decisions. In fact, there was a more negative evaluation of the framed attribute both when the attribute was the described person's blood cholesterol level and when the attribute was the described person's vitamin consumption level.

Despite consistent results across experiment with regard to the direction of the framing effect, it should be noted that the effect size was smaller than in earlier studies of the "standard" attribute framing effect, as the main effect of the framing valence was only marginally statistically significant in each experiment. The framing manipulation was more subtle in the present study than in the earlier studies. Whereas stimulus labels themselves invoked positive or negative associations for terms like "success rate" and "failure rate," subjects in the present study had to infer that being below a designated high risk level is a more positive state than being above a designated low risk level and that being above a designated low protection level is a more positive state than being below a designated high protection level. Some subjects may have made these inferences and others not. (The ones who did not were in fact acting more rationally.)

Nevertheless, the present results show that even this more subtle form of framing may have significant implications. Food choices may well be different for those whose concern about cholesterol is raised by virtue of assessing their own level with respect to a low standard as compared to a high standard. As we showed, vitamin intake is also affected by whether assessment of one's own behavior is referred to a high standard or a low standard. Purchase of health foods, diet programs and exercise programs, and seeking medical treatment are additional examples of behaviors with important economic consequences that might be influenced by subtle changes in the standards by which we assess our current health status. 


\section{References}

1. Levin, I. P., \& Gaeth, G. J. (1988). "How consumers are affected by the framing of attribute information before and after consuming the product." Journal of Consumer Research, 15, 374-378

2. Levin, I. P., Gaeth, G., Schreiber, J. L., \& Lauriola, M. (2002). "A new look at framing effects: Distribution of effect sizes, Individual Differences and Independence of Types of Effect." Organizational Behavior and Human Decision Processes, 88, 411- 429.

3. Levin, I. P., Schneider, S. L., \& Gaeth, G. (1998). "All frames are not created equal: A typology and critical analysis of framing effects." Organizational Behavior and Human Decision Processes, 76, 149188.

4. McNeil, B., Pauker, S. G., Sox, H. C., \& Tversky, A. (1982). "On the elicitation of preferences for alternative therapies." New England Journal of Medicine, 306, 1259-1262.

5. Tversky, A., \& Kahneman, D. (1981). "The framing of decisions and the psychology of choices." Science, 211, 453-458.

6. van Schie, E. C. M., \& van der Pligt, J. (1995). Influencing risk preference in decision making: The effects of framing and salience.” Organizational Behavior and Human Decision Processes, 63, 264-275.

Notes 
Notes 